\title{
Family medicine in Ukraine: changing theory into practice and completing the circle
}

\section{INTRODUCTION}

\author{
Hare: 'Are we going around in \\ circles?' \\ Tortoise: 'Well, that's a good \\ question!' \\ Hare: 'If I didn't ask it, it wouldn't be a \\ question.' \\ Tortoise: 'And it must be a good \\ question if you bothered to ask it!' \\ Hare: 'Are we going around in \\ circles?'
}

The last two and a half decades have seen major changes in health care within Eastern Europe and the previous Soviet states, particularly in their attitude to primary care. ${ }^{1-3}$ Just as the UK is now talking about the emergence of polyclinics to cope with the pressures of primary care, ${ }^{4}$ these countries have abandoned, or are abandoning the polyclinic concept. Health care, which had previously been founded upon consultations with highly specialised consultants, is now moving towards a general practice/family medicine-led service in community-based, integrated clinics. $^{5}$

Although the first experience with a primary care system in the region began in Russia in the $1980 \mathrm{~s},{ }^{6}$ major changes in the former states did not occur until the mid1990s. ${ }^{7.8}$

Ukraine was one of these former Soviet

Box 1. Defining a generalist.

'The personal doctor can offer care which is accessible, of broad range, relatively continuous, and above all, integrative. Not one of these characteristics is easily provided by a specialist; in combination they can only be provided by a generalist. This forms a distinctive role which people value as a basis to which specialist care can be added when necessary. In this way specialists and generalists can relate to each other, not as two people doing the same job, one better than the other, but as two people doing complementary jobs of equal status within the profession and society more widely.'

\author{
Box 2. Major achievements in Ukraine family medicine. \\ - More than 7000 trained family doctors \\ - Nineteen academic Departments of Family Medicine \\ Vocational training scheme \\ - More than 5000 family medicine clinics/ambulatories \\ - Ukraine Association of Family Medicine \\ - Annual Conference of Family Medicine; full congress every 4 years \\ - Highly-ranked scientific Journal of Family Medicine \\ - Individual patient registration in some clinics \\ - Electronic patient records in some clinics \\ - Family medicine presence in undergraduate medical education \\ - Multiprofessional teams in some clinics \\ - Concept of further development of family medicine in Ukraine until 2114 approved by \\ Government in 2008
}

states that agreed to rationalise its health care, using a model described ably by John Horder (Box 1), ${ }^{9}$ assisted by experienced UK GPs and ably supported by the Royal College of General Practitioners. ${ }^{10,11}$

To date, major achievements have been made by the proportionally small number of Ukraine family doctors (Box 2), very often against strong opposition from secondary care specialists and with intermittent support from the Ministry of Health who still remain only semiconvinced as to family medicine's future; however the theory is in place.

Although there have been several significant developments in family medicine, there are still obstacles to be overcome (Box 3). Some of them are of major importance and at least four are inhibitory to further developments:

- family medicine is still not recognised as a separate specialty either among its peers in secondary care or within government circles; and is still not recognised as a scientific specialty which makes it difficult to establish teaching and research;

- some specialties, relevant to family practice remain 'protected' from training programmes by their specialist groups;

- despite family doctors being trained in 'vocational training schemes', the financial resources for teacher training are almost absent; and

- poor financial reward leads to many trained family doctors returning to hospital specialties or leaving medicine and health care completely.

These problems have led to an active healthcare provider who, within a developing organisation and despite seeing and managing patients, provides a less than comprehensive service with patients still wishing referral to secondary care, and a very disillusioned workforce; the theory is in place but practice does not support it. Personal observation suggests that little has changed in a majority of new practices since the reported activity of 1999. ${ }^{11}$

\section{CHANGING THEORY INTO PRACTICE}

In order to be able to influence the appropriate authorities with the idea that family medicine is capable of affecting population health care, two specific 
activities were developed. These are described below, together with their purpose, their intended outcomes, and areas of potential influence.

\section{Workshop developing clinical guidelines}

In a country that is second only to Russia in size within Europe, but a population that is less than the UK (46.7 million) $)^{12}$ it still remains very difficult to obtain true health statistics from Ukraine. However, in a population where $19.4 \%$ of females and $7.4 \%$ of males are clinically obese (BMI $>30 \mathrm{~kg} / \mathrm{m}^{2}$ ) and $60 \%$ of deaths are linked to cardiovascular or cerebrovascular problems,$^{13}$ it is hardly surprising that the chronic conditions of diabetes and hypertension rank high in the population's morbidity figures. Personal observation suggests that although these conditions are actively treated, the wide variation in treatment protocols is driven by the pharmaceutical companies rather than any evidence base and it is accepted as a very cost-inefficient system. Major inroads into a cost-effective and morbidity lowering service could be theoretically possible with the introduction of clinical guidelines based upon evidence-based medicine and practice.

A 2-day course was arranged for May 2007 which brought together specialist cardiologists and endocrinologists from the secondary sector and family doctors, together with representatives from the
Ministry of Health. Instruction was given on Clinical Guidelines, the concept of SIGN ${ }^{14}$ and NICE, ${ }^{15}$ and evidence-based health care and its implementation into UK family practice. Ukraine family doctors and officials from the Ministry of Health spoke about the present Ukraine health situation and its current prescribing policies and statistics. The majority of the workshop was arranged as a small group, with interprofessional discussion on the development of two potential guidelines; management of adult hypertension and management of type 2 diabetes mellitus. It was hoped that this workshop would:

- standardise the management of both conditions;

- further support the development of effective health and cost-effective care in family medicine;

- stimulate shared work between the primary and secondary care sectors;

- demonstrate to the ministry that this approach is paramount to family medicine and the present cadre of family doctors can deliver it effectively; and

- promote family medicine as an efficient and effective service.

\section{Workshop on developing a youth friendly service}

From a worldwide perspective, there are factors that place today's adolescents in a heightened position for poor health outcomes: society's changing expectations,

\section{Box 3. Obstacles to the further development of Ukraine family medicine.}

- Family medicine is still not recognised as a scientific specialty, not allowing for recognised teaching and research within universities

- Lack of support for core specialties in vocational training (paediatrics, gynaecology)

Opposition from majority of secondary care sector

- Poor financial support for training

- Extremely low salaries

Trained family doctors leaving the service

Absence of national curricular for undergraduate training

Absence of documented core competencies

Lack of trainers and training practices

- Low level of status of professional associations

Poor acceptance of family medicine by population, especially in cities changing behaviours of adolescents, and peer pressure. Health trends among the youth of Ukraine reflect the pressures that their global neighbours share. Ukraine is within the top five European countries for teenage abortion, where only $2.8 \%$ of fertile females use hormonal contraception. ${ }^{16}$ Ukraine is experiencing one of the fastest growing HIV epidemics in the world, 1.4\% of people aged between 15 and 49 years are HIV positive, (known to be a gross underestimate) and $45 \%$ of new cases are found in teenage drug abusers. ${ }^{17}$ Although youth friendly clinics have been developed, opinion suggests that they are not effective, especially in dealing with the mental health problems of Ukraine youth ${ }^{18}$ and many believe a more suitable positioning for these services would be in primary care.

A 4-day, interprofessional course, coorganised with the regional office of the World Health Organisation (WHO), was created, following the guidelines workshop dealing specifically with how a youth friendly health service could be rolled out within the family medicine clinics in Kiev. A roll-out over the whole of Ukraine was considered too large a project initially, either to manage or evaluate. Key members from the Ukraine Ministry of Health's Youth Services were present and active in discussions. If successful it was hoped that this course would:

- create an acceptable youth friendly service within family practice;

- create a friendly environment, able to deal with major health issues within the youth of Kiev;

- create opportunity and data for effective community-based research; and

- demonstrate that family practice is the correct environment to deliver a youth friendly service.

\section{RESULTS}

The purpose of this exercise was to gauge the interest of the participants in both topics, and to assess whether the topics could be used to further promote the value and effectiveness of family medicine. A quantitative questionnaire of evaluation was thought unnecessary at this stage. Due to the interprofessionality and some of the language difficulties, a qualitative 
exercise using post-course small group discussion led by two of the authors was seen to be an appropriate method of evaluating the workshops and judging the potential of the activity.

\section{Workshop on the development of clinical guidelines}

Although there was some initial difficulty in understanding the concepts of evidencebased health care, protocols, and clinical guidelines, these were eventually understood by all participants who agreed that this was a logical step forward within family medicine. A particularly positive response was received from the representatives of the Ukraine Ministry of Health, who perceived the concept as a way of reversing or eliminating the financially adverse effects of the pharmaceutical industry in influencing prescribing.

Evidence-based medicine and evidencebased health care was very well received by primary and secondary healthcare providers alike; the workshop saw the emergence of two interprofessional working groups, who were willing to embark upon the process of developing two clinical guidelines, the progress of which will be presented at the October 2008 Conference of Ukraine Family Medicine.

\section{Workshop on developing youth friendly services}

The workshop groups discussed the present activities of the existing 40 adolescent clinics. However, the local health workers, including those who had worked in the clinics, considered that they were not as well attended as they could have been, or as purposeful as hoped. The delivery of such a service by the new family medicine clinics was accepted unanimously by the participants and by the Ministry of Health representatives for Youth Services. The conclusion was reached in which the WHO regional office would work with the Ministry of Health and the Ukraine Association of Family Medicine and run a pilot scheme within the Kiev Region in developing the service and evaluating it through its organisers and customers. This work will also be presented at the October 2008 Conference on Family Medicine.

\section{DISCUSSION}

The process of family medicine in Ukraine began in 1987 when a group of Russian physicians visited Lviv, Western Ukraine, to talk about the concept. ${ }^{19}$ However, it was not until the post-Soviet era in the early 1990s that the idea was truly accepted by the Ukraine government and began to gain momentum and provide the tremendous changes that were discussed earlier (Box 2). Interestingly enough, and despite some very major changes, similar obstacles described in $1987^{19}$ are still present in Ukraine to this day; that is, the finances to underpin the service, the payment of the practitioners, the acceptance of the specialty and its practitioners in the eyes of the government and secondary care, and an evaluation/assessment process of the service.

Many of the former Soviet countries accepted the Alma Ata Declaration of $1978^{20}$ and were happy to see it incorporated into the European Health for all Policy of 1998. ${ }^{21}$ Both have given credibility to the development of family medicine.

Although papers have been written about the various initiatives in Eastern Europe, very little evidence is emerging about the true effects of family medicine upon the health care of the country. Changes in some health statistics are improving, people are tending to live longer, and management of long-term conditions appears to be improving, but issues such as the increasing termination of pregnancy rate and the HIV explosion ${ }^{15}$ still dominate: surely areas that family medicine was supposed to address and improve through its bio-psycho-social approach to health care.

Over the many years of developing family medicine in Ukraine, it is the authors' opinions that although family medicine is present, a longitudinal approach to its development predominates. This approach allows some change to occur, but the lack of any evaluation or research, which then informs further development in a cyclical manner, limits its usefulness.

Family medicine in Ukraine, although accepted at government level, still has failed to get full recognition, either by the
Ministry or secondary care colleagues. As such it remains a 'Cinderella' subject; poorly paid and poorly supported. Many specialists, paediatricians, obstetricians and gynaecologists still fail to recognise it as a subject and refuse to be involved with any form of vocational training (although this cannot be said to be universal, there are pockets of very strong support); hence mothers still have little confidence in the family doctor in dealing with pregnancy and their children.

The salaries of family doctors are lower than the exceptionally low salaries of other specialists. Fifty per cent of trained family doctors leave the service on a yearly basis, often pursuing careers in more lucrative work such as the pharmaceutical industry or even in the retail industry.

This short piece of work in Ukraine represents a new step in trying to bring a cyclical approach to the management of change; a different approach that will attempt to convince the government that family medicine is a viable option for effective health care. It will of course hopefully stimulate a debate about a restructuring of the pay scales for the family doctors and community health workers.

Although the two workshops described were not major pieces of communitybased research, they will hopefully give rise to a much more structured long-term evaluation and lead to further research on the subjects.

The future of Ukraine family medicine is bright; it is moving forward. This next stage of its development will hopefully develop it even further.

\section{Trevor Gibbs, Lyudmila Khimion, Grygorii Lysenko}

\section{Funding}

This work was originally supported by the International Committee of RCGP. It is now supported through the Ukraine Travel Fellowship of RCGP-Scotland

\section{Acknowledgements}

Without the support from its energetic and passionately supportive family doctors this initiative would not be in existence; acknowledgement is not enough but is all we can offer at present. Special thanks are given to Valentina Baltag and Anastasiya Dumcheva from the World Health Organisation. 


\section{REFERENCES}

1. Ryan M, Stephen J. General practitioners and family doctors in the Russian Federation. Br J Gen Pract 1996; 46: 487-489

2. Earl-Slater A. Health care reforms in the Czech Republic. J Manag Med 1996; 10: 13-22.

3. Toon P. Reforming the Russian Health Service. BMJ 1998; 317: 741-744.

4. Healthcare for London. Consulting the capital. London, 2007.

http://www.healthcareforlondon.nhs.uk/background. asp (accessed 8 Aug 2008).

5. Rese A, Balabanova D, Danishevski K, McKee M. Implementing general practice in Russia: getting beyond the first steps. BMJ 2005; 331: 204-207.

6. Ministry of Health of Russian Federation. Decree \# 237: Stepwise transition of outpatient services to general practice model. Moscow: 1992

7. Danishevski K, Balabanova D, McKee M, Atkinson S The fragmentary federation: experience with the decentralized health system in Russia. Health Policy Plan 2006; 21:183-194.

8. Fister K, McKee M. Health and healthcare in transitional Europe. BMJ 2005; 331: 169-170.

9. Horder J. The College in my practice. Br J Gen Pract 1992; 42: 126-128.

10. Gibbs T, Mulka O, Zaremba E. The Royal College of General Practitioner's Fellowship Scheme 1993-1997. Eur J Gen Pract 1998; 4: 84-87.

11. Gibbs T, Mulka O, Zaremba E, Lysenko G. Ukrainian general practitioners: the next steps. Eur I Gen Pract 1999; 5: 33-36.

12. Culture of Ukraine.

http://www.everyculture.com/To-Z/Ukraine (accessed 4 Aug 2008)

13. World Health Organisation. The European health report 2005. WHO: Geneva, 2005. http://www.euro.who.int/ehr2005 (accessed 8 Aug 2008)

14. Scottish Intercollegiate Guideline Network. NHS Quality Improvement Scotland, 2001. http://www.sign.ac.uk (accessed 4 Aug 2008).

15. National Institute for Health and Clinical Excellence. NICE: London, 2007. http://www.nice.org.uk (accessed 4 Aug 2008)

16. Vovk I. The importance of family planning in Ukraine. In: Johannisson E, Kovacs L, Resch BA, et al (eds). Assessment of research and service needs in reproductive health in Eastern Europe - concerns and commitments. New York, Parthenon Publishing Group, 1997: 217-218.

17. Alliance. Supporting community action on AIDS in Europe. http://www.aidsalliance.org (accessed 4 Aug 2008).

18. Godlevsky AG. Youth mental health and development of community mental healthcare services in Ukraine. European Psychiatry 1998; 13(4): 250.

19. Furtak I. A beginning of family medicine practice in Ukraine. Annu Meet Int Soc Technol Assess Health Care Int Soc Technol Assess Health Care Meet. 1997; 13: 150.

20. World Health Organisation and UNICEF. Primary health care: report of the International Conference on Primary Health Care, Alma-Ata USSR. Geneva: WHO, 1978.

21. World Health Organisation. Health for all in the 21st century. Geneva: World Health Organisation, 1998

DOI: 10.3399/bjgp08X342066

\section{The call of general practice beckons}

The time of the year when our health service was at greatest risk of being thrown into chaos has thankfully passed. Newly-qualified interns have commenced work as doctors for the first time, fresh from that blissful 6 weeks between passing final medical exams and starting the new job. Short straws were drawn, meaning some of them started their careers on call at a weekend; always a dreaded prospect which at least a quarter of us have experienced. In addition to this, newly-appointed registrars in all specialties took up new posts with considerably more responsibility. This situation coupled with the skeletal or sometimes nonexistent ancillary medical services at weekends, particularly in the smaller hospitals all adds to the stress. My colleagues and I in the third year of the Western Area GP training programme have left all this behind, no more bleeps, no more continuous 36-hour shifts every fourth or fifth night and no more having to be in two places at once. Yes, we have left the rocky terrain of hospital life for the greener pastures of general practice.

The popularity of a career in general practice has increased enormously over the past few years. This is clearly reflected in the strong competition for places on the vocational training programmes. Most interns now wish to pursue this specialty where as recently as 4 years ago only half of my graduating class were even considering it. The appeal of life as a GP has also increased considerably over the past decade. General practice has revolutionised itself in many ways. The organisation of out-of-hours cooperatives and the appointment system are probably the most notable. All of this has enhanced the appeal of working in the community and providing continuity of care. The really fantastic feature of general practice is that it is all about interacting with people and building lifelong relationships that cross generations. Most interaction in a hospital is between staff whereas the opposite is the case in the community. The variety of medical issues that arise in general practice simply cannot be matched in any other specialty, from signing certs to thromblysing in the community means no 2 days are the same. The vast majority of medical consultations in Ireland take place in GP surgeries and most medical conditions are successfully managed in this setting.

My colleagues and I have completed 2 years of hospital posts in addition to our intern year. The rotation consists of psychiatry, A\&E, obstetrics and gynaecology, medicine, and paediatrics, all very necessary for working in the community. The pace of life in Irish hospitals, particularly the smaller ones has increased enormously over the past number of years, the demands placed on them particularly in the winter months are huge. Most of us will not miss the long shifts with little or no sleep. It was with a light heart I turned in my bleep to the busy hospital switchboard receptionist, never to hold it again, that small but loud creature that resided on my belt for the past 6 months whose shrill wail is indelibly marked on my brain forever, its call guaranteed to increase blood pressure and heart rate simultaneously. The European Working Time Directive has largely remained aspirational in the smaller hospitals. The workload, particularly when on call is likely to remain onerous for the foreseeable future.

It has not been all negative in the hospital posts. We will all miss the camaraderie of other colleagues and the whole social aspect to being a member of hospital staff; there was always some excuse for a night out. I suspect we will find working alone, particularly in the out-of-hour's situation, a big challenge. In the hospital there is always somebody to consult if in doubt. Some of us may find working in rural areas lonely and emergencies in this setting can be truly stressful especially if you are the only doctor for a 30 mile radius. It is hard to beat the small country hospital in terms of learning because you are at the coalface when on call. There is no quicker way to learn than on your feet in an emergency situation. I have had the privilege of working with some truly dedicated, brilliant people who were always willing to teach.

Overall the hospital experience has been great, particularly in terms of learning. We have emerged battle hardened and hopefully have gained adequate experience to serve our patients in the community well.

\section{Robert Marsh}

DOI:10.3399/bjgp08X342075 